\title{
Elucidating the formation and structural evolution of platinum single-site catalysts for hydrogen evolution reaction
}

\author{
Peng Tang ${ }^{1}$, Hyeon Jeong Lee ${ }^{1}$, Kevin Hurlbutt ${ }^{1}$, Po-Yuan Huang ${ }^{1}$, Sudarshan \\ Narayanan $^{1}$, Chenbo Wang ${ }^{2}$, Diego Gianolio ${ }^{5}$, Rosa Arrigo ${ }^{6}$, Jun Chen ${ }^{1}$, Jamie H. \\ Warner $^{3,4}$, and Mauro Pasta ${ }^{1,2}$ \\ ${ }^{1}$ Department of Materials, University of Oxford, Parks Road, Oxford OX1 3PH, United \\ Kingdom \\ ${ }^{2}$ Oxford Suzhou Centre for Advanced Research, 388 Ruoshui Road, Suzhou 215123, \\ Jiangsu Province, P. R. China \\ ${ }^{3}$ Materials Graduate Program, Texas Materials Institute, The University of Texas at \\ Austin, 204 East Dean Keeton Street, Austin, Texas, 78712, United States \\ ${ }^{4}$ Walker Department of Mechanical Engineering, The University of Texas at Austin, 204 \\ East Dean Keeton Street, Austin, Texas, 78712, United States \\ ${ }^{5}$ Diamond Light Source Ltd., Harwell Science and Innovation Campus, Chilton, Didcot, \\ OX11 0DE, UK \\ 6 School of Science, Engineering and Environment, University of Salford, M5 4WT \\ Manchester, U.K
}

\begin{abstract}
Platinum single-site catalysts (SSCs) are a promising technology for the production of hydrogen from clean energy sources. They have high activity and maximal platinum-atom utilisation. However, the bonding environment of platinum during operation is poorly understood. In this work, we use operando, synchrotron-X-ray absorption spectroscopy to study the platinum bonding in SSCs. First, we synthesise an atomically dispersed platinum complex with aniline and chloride ligands onto graphene and characterise it with ex-situ electron microscopy, X-ray diffractometry, X-ray photoelectron spectroscopy, X-ray absorption near edge structure spectroscopy (XANES), and extended X-ray absorption fine structure spectroscopy (EXAFS). Then, by operando EXAFS and XANES, we show that as a negatively biased potential is applied, the Pt-N bonds break first followed by the Pt-Cl bonds. The platinum is reduced from platinum(II) to metallic platinum(0) by the onset of the hydrogen-evolution reaction at $0 \mathrm{~V}$. Furthermore, we observe an increase in Pt-Pt bonding, indicating the formation of platinum agglomerates. Together, these results indicate that while aniline is used to prepare platinum SSCs, the single-site complexes are decomposed and platinum agglomerates at operating potentials. This work is an important contribution to the understanding of the bonding environment and the evolution of the molecular structure of platinum complexes in SSCs.
\end{abstract}

\section{Introduction}

Catalysts for the electrochemical hydrogen evolution reaction (HER) are desirable to be able to produce hydrogen from clean sources like solar energy. [1, 2] Platinum is among the most active HER catalysts known in acidic media. [3] Platinum is a heterogeneous catalyst, with the HER occurring only 
on the metal surface.[4] This can be prohibitively inefficient because platinum is so rare and expensive. [5]

Single-atom catalysts (SACs) are systems in which electrocatalytically active metal atoms are loaded onto a suitable substrate and separated from any other metal atoms by a distance larger than their metallic bonding. [6, 7] They have extremely high atom utilisation because every catalytic atom is exposed to the reactants and available for reaction. [8, 9]

Platinum SACs can be prepared by synthesising a platinum complex and adsorbing the whole complex to a suitable substrate. [10-12] We choose to distinguish these structures and refer to them as single-site catalysts (SSCs). This is to emphasise that these structures have well-separated sites of catalysis, but, as complexes, they are composed of multiple atoms. [13-15]

Despite the promise of platinum SSCs for the HER, the bonding environment of platinum in these devices before and during operation is poorly understood. [16-19] Establishing the structure-property relationship between the platinum bonding and the electrochemical performance of the catalyst is critically important to enabling design of tailored, high performing platinum SSCs. [20, 21]

In this work we examine the model SSC system of a platinum complex with aniline and chloride ligands adsorbed onto graphene. Graphene is an excellent substrate because of its high electronic conductivity, chemical stability, [22] and the good contrast it provides with platinum in electron microscopy. [23] At the same time, aniline is stabilised on adsorption onto graphene through pi-pi stacking interactions with the molecule's aromatic ring. [10] Furthermore, aniline readily complexes with platinum and the molecule's large size provides a steric hindrance that can prevent platinum agglomeration. [24, 25]

We characterise the morphology and physical structure of the as-synthesised SSC with ex-situ scanning transmission electron microscopy (STEM) and X-ray diffractometry (XRD). We examine the SSC's oxidation state and bonding environment with photoelectron spectroscopy (XPS), X-ray absorption near edge structure spectroscopy (XANES), and extended X-ray absorption fine structure spectroscopy (EXAFS). Finally, we use chronoamperometry with operando, synchrotron EXAFS and XANES to study how the platinum's bonding environment and coordination number change as a function of applied potential during the HER. This work is an important contribution to the understanding of the synthesis and stability of platinum SSCs.

\section{Results and Discussion}

\section{Synthesis and physiochemical characterisation}

The platinum SSC is prepared according to a modified impregnation method described in detail in the Experimental procedures section and outlined in Figure 1a.

High-angle annular dark-field scanning transmission electron microscopy (HAADF-STEM) of (Pt/Ani/Ar) and (Pt/Ar) are shown in Figure 1c-d and e-f, respectively. (Pt/Ani/Ar) contains atomically dispersed platinum with an average areal number density of about $0.5 \mathrm{~nm}^{-2}$ (Supplementary Figure S2a). Some clusters of platinum are present;[25] these are generally of size $1 \mathrm{~nm}$ or smaller (Supplementary Figure S2a). (Pt/Ar) mainly contains larger nanoparticles that are up to about $4 \mathrm{~nm}$ across (Supplementary Figure S2b).

The XRD patterns for the glassy carbon (GC) electrode and for (Pt/Ani/Ar) are shown in Figure 1b. [26] The two patterns match well. In particular, the pattern for (Pt/Ani/Ar) lacks the intense peaks at about $39.8^{\circ}, 46.2^{\circ}$, and $68.5^{\circ}$ expected for the (111), (200), and (220) reflections in crystalline platinum (PDF\#04-0802). This implies that the HAADF-STEM images, which show no platinum nanoparticles in $(\mathrm{Pt} / \mathrm{Ani} / \mathrm{Ar})$, are representative of the bulk sample. The STEM images show that treatment with aniline promotes the formation of more atomically dispersed platinum.

\section{Ex-situ XPS and XAS}

The platinum oxidation state is investigated by XPS, and the spectra for $(\mathrm{Pt} / \mathrm{Ani} / \mathrm{Ar})$ and $(\mathrm{Pt} / \mathrm{Ar})$ are shown in Figure 2a. Two standards are also shown: ammonium hexachloroplatinate(IV) $\left(\left(\mathrm{NH}_{4}\right)_{2} \mathrm{PtCl}_{6}\right)$ and platinum(II) chloride $\left(\mathrm{PtCl}_{2}\right)$. The platinum $4 \mathrm{f}_{7 / 2}$ peak in $\mathrm{PtCl}_{2}$ has a binding energy of 72.9 $\mathrm{eV}$ consistent with the +2 oxidation state. The $\left(\mathrm{NH}_{4}\right)_{2} \mathrm{PtCl}_{6}$ has a higher binding energy of 75.2 $\mathrm{eV}$, which is also expected for its high oxidation state of +4 . The spectrum for $(\mathrm{Pt} / \mathrm{Ani} / \mathrm{Ar})$ is well fitted by a $74 \%$ contribution from a peak centred 

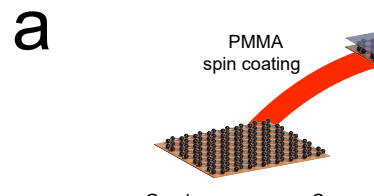

Graphene grown on $\mathrm{Cu}$

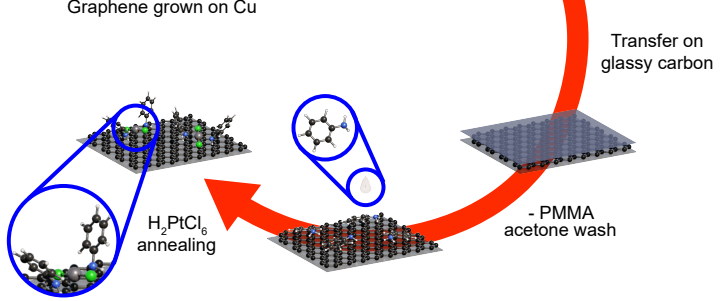

b

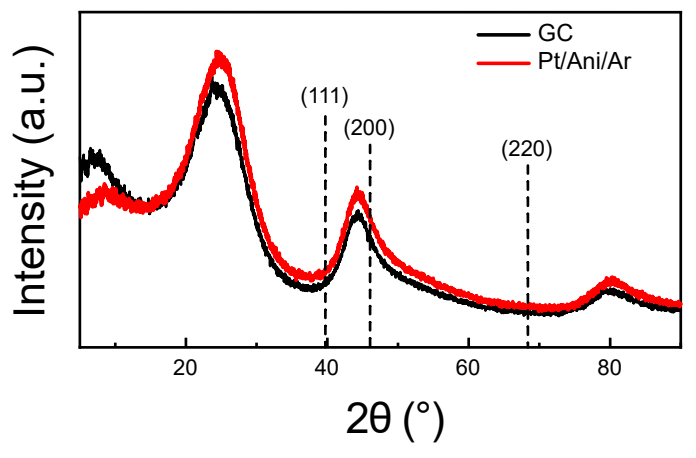

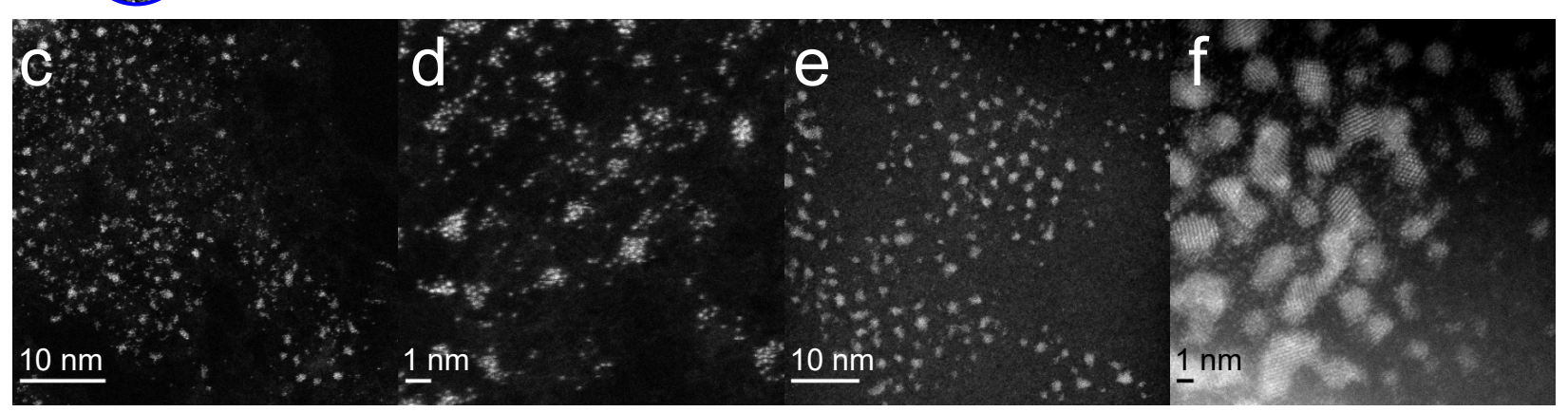

Figure 1. a, Schematic illustration of the synthesis process (5 steps) for Pt SSC (grey, Pt; green, Cl; blue, N; black, carbon; white, H). Step 1: graphene nanosheets are grown by chemical vapour deposition (CVD) on copper; Step 2: copper foil is etched after spin-coating with polymethyl methacrylate (PMMA) on top; Step 3: Thin-layer graphene coated with PMMA is then transferred onto a polished glassy carbon (GC) electrode; Step 4: after washing the PMMA with acetone and heating to $250{ }^{\circ} \mathrm{C}$ to remove trace organic contaminants, aniline is drop-cast onto the graphene-coated GC electrode and dried; Step 5: chloroplatinic acid in ethanol is then drop-cast and annealed under argon atmosphere. This sample is referred to as the (Pt/Ani/Ar) sample. The control sample (Pt/Ar) is prepared by the same method but without aniline. $\mathbf{b}$, X-ray diffraction patterns for pristine GC and a (Pt/Ani/Ar) sample on GC; low magnification to high magnification atomic resolution HAADF-STEM images of (Pt/Ani/Ar) (c, d) and $\mathrm{Pt} / \operatorname{Ar}(\mathbf{e}, \mathbf{f})$.

at $72.8 \pm 0.1 \mathrm{eV}$ and a $26 \%$ contribution from a peak centred at $74.6 \pm 0.1 \mathrm{eV}$ (Figure $2 \mathrm{a}$ and Supplementary Figure S6b). These contributions likely arise from platinum(II) and platinum(IV), [27] respectively, indicating that the sample is a mixture of the two oxidation states. $[20,21,28]$ The $(\mathrm{Pt} / \mathrm{Ar})$ is well fitted by a single peak centred at $73.0 \pm$ $0.1 \mathrm{eV}$ arising from the +2 oxidation state. [29, 30] These oxidation-state assignments are consistent with the XANES spectra shown in Figure 2b. Here, reference spectra for $\mathrm{PtCl}_{2}(+2)$ and $\left(\mathrm{NH}_{4}\right)_{2} \mathrm{PtCl}_{6}$ $(+4)$ have white line peak positions at $11565.8 \mathrm{eV}$ and $11567.7 \mathrm{eV}$, respectively. The spectrum for (Pt/Ani/Ar) has a single peak centred at $11566.7 \mathrm{eV}$. This is expected of a mixture of platinum(II) and platinum(IV) and is consistent with the XPS data.

\section{$[28,29]$}

EXAFS spectra plotted in Figure 2c (along with those of the standards) give information about the platinum bonding environment. The standard samples of $\mathrm{PtCl}_{2}$ and $\left(\mathrm{NH}_{4}\right)_{2} \mathrm{PtCl}_{6}$ have one dominant peak in the range of 1.5 to $2.3 \AA$ with the centre at $1.96 \AA$, suggesting that this peak can be associated with $\mathrm{Pt}-\mathrm{Cl}$ bonding. The most intense peak in the spectrum for (Pt/Ani/Ar) is at $1.96 \AA$ with a shoulder at $1.66 \AA$. The peak at $1.96 \AA$ should arise from the presence of $\mathrm{Pt}-\mathrm{Cl}$ bonds. This shoulder peak at $1.66 \AA$ is indicative of platinum-nitrogen bonding. [31, 32] Finally, there is absence of obvious Pt-Pt bonding at $2.73 \AA$ (green curve in Figure 2c), which also suggests that the platinum atoms are mainly isolated from each other. We therefore 

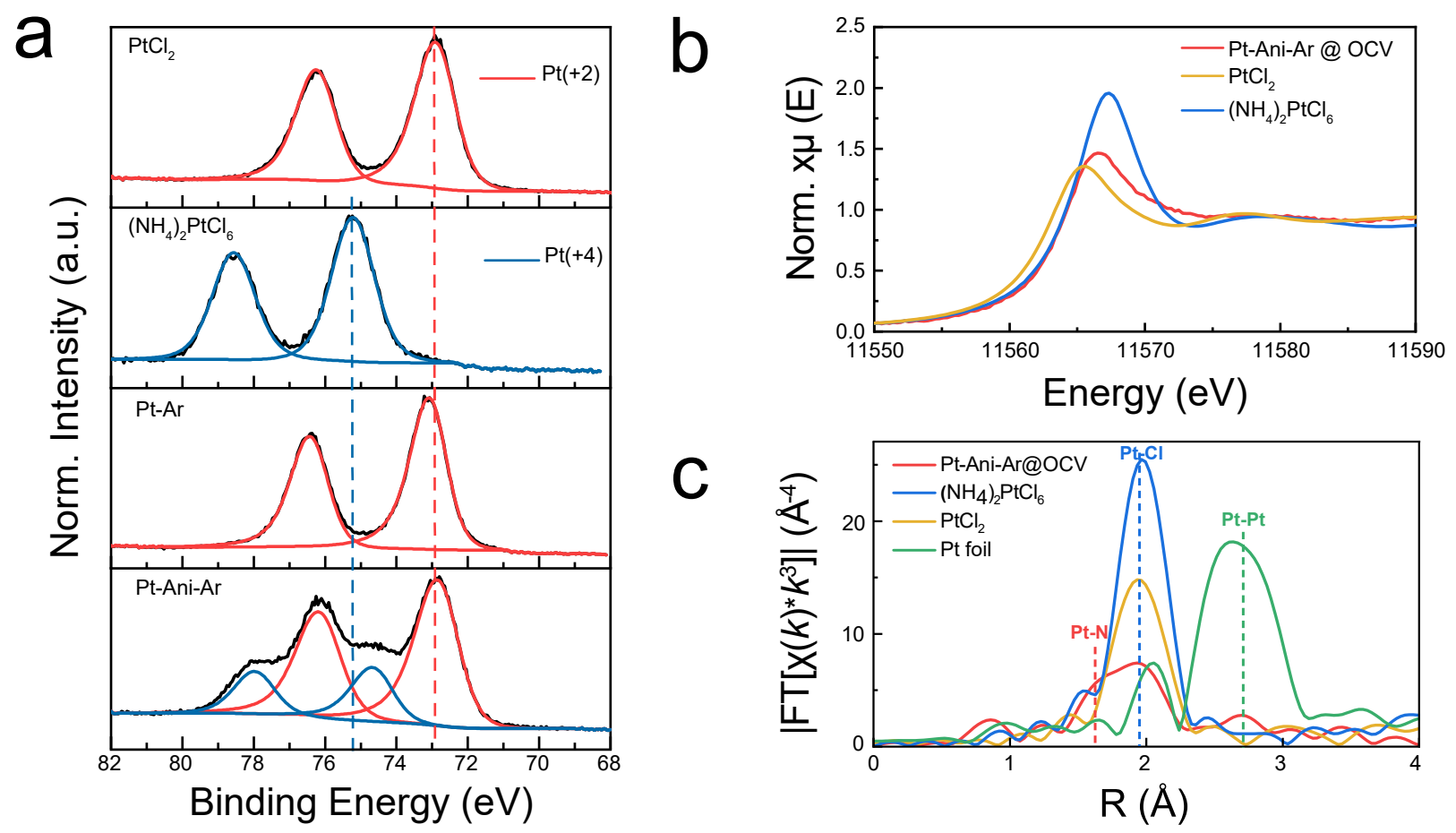

Figure 2. a,b, XPS spectra of Pt $4 \mathrm{f}(\mathbf{a})$ and XANES spectra (b) recorded at $\mathrm{Pt}_{3}$-edge for the synthesised Pt SSC and control sample (Pt/Ar) compared with reference standards in the oxidation states of platinum(II) and platinum(IV). The vertical dashed red and blue lines in (a) indicate the peak positions of $\mathrm{Pt}_{7 / 2}$ at the oxidation state of platinum(II) and platinum(IV). c, Corresponding $\mathrm{k}^{3}$-weighted Fourier-transformed $\mathrm{Pt} \mathrm{L}_{3}$-edge EXAFS spectra of samples in (b) and standard $\mathrm{Pt}$ foil. The vertical dashed lines show the positions of Pt-N (red), Pt-Cl (blue), and Pt-Pt (green) peaks.

interpret these data to mean that the aniline and chloride coordinate to the platinum to form a complex molecule.

These XPS and XANES data indicate that $(\mathrm{Pt} / \mathrm{Ani} / \mathrm{Ar})$ and $(\mathrm{Pt} / \mathrm{Ar})$ are reduced in situ from platinum(IV) (the oxidation state of platinum in the precursor $\mathrm{H}_{2} \mathrm{PtCl}_{6}$ ) to platinum(II). This is likely caused by the ethanol solvent (Supplementary Figure S5). [33] However, in (Pt/Ani/Ar) the platinum forms a complex with the aniline (as confirmed by the bonding data from the EXAFS spectra) which decreases the platinum reduction potential. This is the reason that $(\mathrm{Pt} / \mathrm{Ani} / \mathrm{Ar})$ is only partially reduced, with platinum(IV) still present. The STEM, XPS, XANES and EXAFS data, suggest that the aniline-treated sample contains atomically dispersed platinum in the form of molecular complexes (Pt single-site complexes) physisorbed onto the graphene substrate.

\section{Operando XAS measurements}

Operando hard XAS at the platinum $\mathrm{L}_{3}$-edge was performed at different potentials to investigate the evolution of the platinum single-site complex's oxidation state and chemical bonding environment during HER. [18, 34, 35] A customised three-electrode cell setup (Supplementary Figure S8) was mounted to investigate the aniline-treated sample (Pt/Ani/Ar). The chronoamperometric performances of (Pt/Ani/Ar) at various potentials are shown in Figure 3a (with a particular shown in $3 \mathrm{~b}$ ). The areal current densities are shown for at least $1000 \mathrm{~s}$ of operation at each of 10 voltages: $0.4,0.3,0.2,0.1,0.06,0.02,0,-0.02,-0.04$, and $-0.06 \mathrm{~V}$ versus the reversible hydrogen electrode (RHE). The open-circuit voltage (OCV) is 0.6 $\mathrm{V}$ versus RHE. None of the tests at a positive potential has an areal current density greater than $0.1 \mathrm{~mA} \mathrm{~cm}{ }^{-2}$. This is to be expected since the HER is only thermodynamically favourable below $0 \mathrm{~V}$ 
versus RHE. Indeed, the data for the test at $0 \mathrm{~V}$ show a minimal areal current density until about $600 \mathrm{~s}$ (out of approximately $1200 \mathrm{~s}$ in total) when the current jumps suddenly and then gradually increases to about $-0.3 \mathrm{~mA} \mathrm{~cm}{ }^{-2}$. The areal current densities for the subsequent potentials of $-0.02,-0.04$, and $-0.06 \mathrm{~V}$ versus $\mathrm{RHE}$ are $-0.5,-0.9$, and -1.5 $\mathrm{mA} \mathrm{cm}{ }^{-2}$, respectively. The Tafel slope, calculated using the 4 negative potentials, is $60 \mathrm{mV} \mathrm{dec}^{-1}$. This is comparable to the Tafel slope of platinum nanoparticles for the HER. [36, 37]

The average of 8 XANES spectra, collected during operation at each potential, are shown in Figure 3c. These data show that the oxidation state of platinum decreases from a mixture of +4 and +2 to 0 . When the potential is decreased from $0.6 \mathrm{~V}$ (OCV) to $0 \mathrm{~V}$ versus RHE, the positions of both the white line (red arrow and right inset in Figure 3c) and absorption edge (left inset in Figure 3c) in the XANES spectra show a negative shift of $0.4 \mathrm{eV}$. Those shifts suggest a continuous decrease of platinum valence from a mixture of platinum(II) and platinum(IV) to metallic platinum. [19, 38]

The bonding environment of the platinum also changes as the applied potential varies, as shown in the EXAFS spectra in Figure 3d. As the potential is decreased from OCV to 0 , the peaks at 1.66 and 1.96 $\AA$, arising from $\mathrm{Pt}-\mathrm{N}$ and $\mathrm{Pt}-\mathrm{Cl}$ bonds, respectively, both decrease in intensity. But the peak arising from Pt-N (blue vertical line) decreases faster than that arising from $\mathrm{Pt}-\mathrm{Cl}$ (orange vertical line). This indicates that as the potential is decreased from OCV and while the oxidation state of the platinum falls to 0 , the aniline molecules are dissociating from the complex. The chloride ions are also dissociating from the platinum atoms, but to a lesser extent compared to the aniline molecules. At $0 \mathrm{~V}$, the SSC has two distinct EXAFS spectra (yellow and olive curves in Figure $3 \mathrm{~d}$ and isolated in Figure S10): one at the low areal current density (-0.1 $\mathrm{mA} \mathrm{cm}{ }^{-2}$ ) before the onset of the HER and one at the higher areal current density $\left(-0.3 \mathrm{~mA} \mathrm{~cm}^{-2}\right)$ after the onset of the HER. Before the onset of the HER, the bonding environment still shows appreciable Pt-Cl bonding and some Pt-N bonding. After $600 \mathrm{~s}$ and the beginning of hydrogen evolution, almost all Pt-N bonding character is lost and the $\mathrm{Pt}-\mathrm{Cl}$ bonding feature decreases significantly. The EXAFS spectrum is instead dominated by $\mathrm{Pt}-\mathrm{Pt}$ bonding. The rapid breaking of $\mathrm{Pt}-\mathrm{N}$ and $\mathrm{Pt}-\mathrm{Cl}$ bonds at $0 \mathrm{~V}$ may be caused by hydrogen molecules aggregated on the SSC.
It has been experimentally shown that the diffusion and agglomeration of platinum atoms can be induced by exposure to a hydrogen atmosphere. [39-41] Since more reduced platinum structures after $600 \mathrm{~s}$ can boost the evolution of hydrogen by lowering the Gibbs free energy of hydrogen adsorption in acidic electrolytes, $[19,42]$ the sudden increase in current density here (discussed in Figure 3a) can probably be justified accordingly.

\section{Structure-electrochemistry correlation}

The previous section elucidates the evolution of the $\mathrm{Pt}$ single-site complex into metallic nanoclusters at potentials below $0 \mathrm{~V}$; the details of this transformation need to be quantitatively examined together with catalytic activity. The effect of applied potential on both areal current density and platinum oxidation state in the SSC is shown in Figure 4a. The height of the XANES normalised white lines is summarised (black plot in Figure 4a) to quantify the evolution of platinum's oxidation state and the electron occupancy of platinum's $5 d$ orbitals. $[43,44]$ The white-line height decreases precipitously from OCV $(0.60 \mathrm{~V})$ to about $0 \mathrm{~V}$ versus RHE. This is consistent with the complete filling of the platinum $5 d$ valence orbitals and the formation of metallic platinum. [19] At the same potential, the corresponding areal current density increases precipitously as the HER becomes thermodynamically favourable, although they are still relatively low (from -0.001 to $-0.08 \mathrm{~mA} \mathrm{~cm}^{-2}$ ). There is an obvious drop in white-line height at around $0 \mathrm{~V}$, and the white-line height stabilises at around 1.30 after $0 \mathrm{~V}$, indicating the oxidation state of platinum remains as 0 at potentials below $0 \mathrm{~V}$. Corresponding to the decrease in oxidation state, a significant increase in current density is also recorded at $0 \mathrm{~V}$ (red plot in Figure 4a). And after the increase, a linear relationship between the base-10 logarithm of the areal current density and the potentials is presented.

EXAFS curve-fitting analysis on the platinum's EXAFS spectrum at OCV allows for the determination of a plausible molecular structure for the platinum complex adsorbed onto graphene. Considering 8 possible platinum complex molecules described by $\left(\mathrm{C}_{6} \mathrm{H}_{5} \mathrm{NH}_{2}\right)_{\mathrm{x}} \mathrm{Cl}_{\mathrm{y}} \mathrm{Pt}$ for $\mathrm{x}=1$ or 2 and $\mathrm{y}=1,2,3$, or 4 , the Pt-N and $\mathrm{Pt}-\mathrm{Cl}$ bond lengths (about 2.05 A and $2.30 \mathrm{~A}$, respectively) determined by density-functional theory modelling of the isolated molecular complexes are the 
a
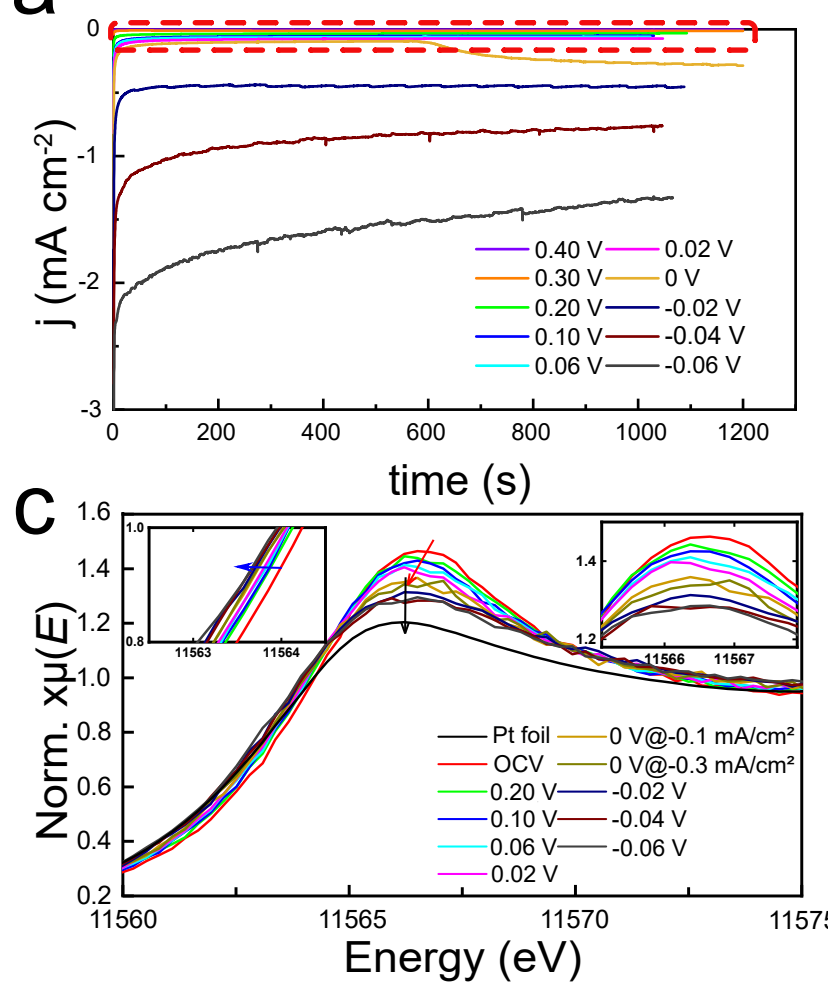

b
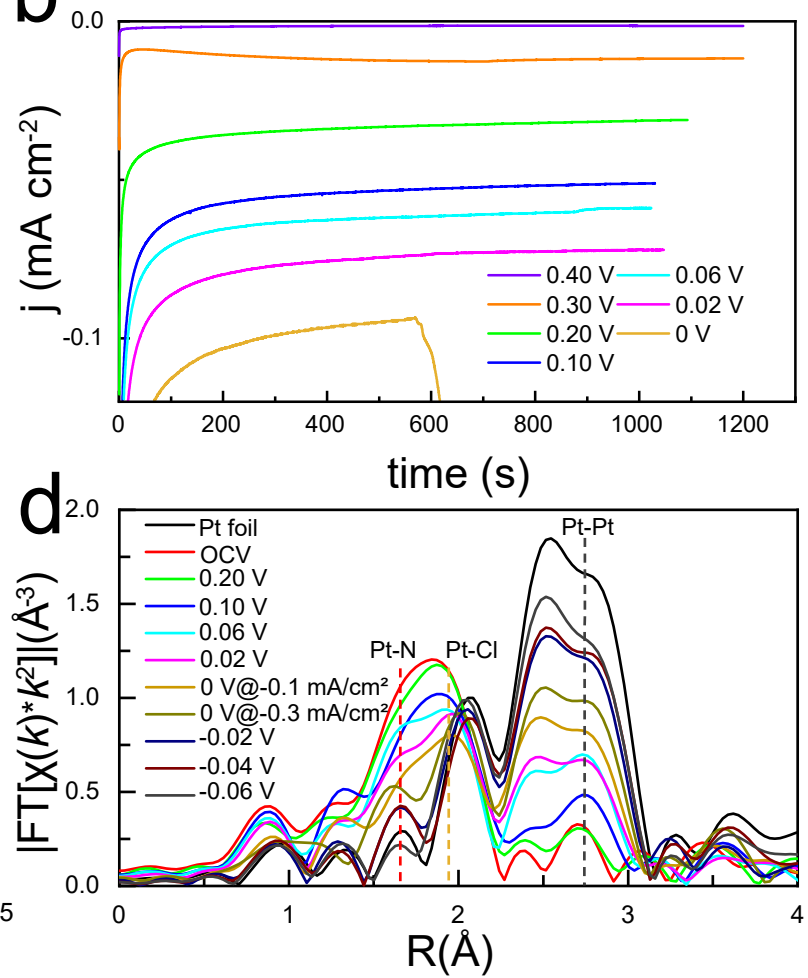

Figure 3. a, Stable chronoamperometric curves of Pt single-site complex at variable applied potentials; b, Enlarged red dashed area in (a) shows the chronoamperometric curves at current density smaller than $0.1 \mathrm{~mA} \mathrm{~cm}{ }^{-2} ; \mathbf{c}$, Operando XANES spectra recorded at $\mathrm{Pt} \mathrm{L}_{3}$-edge of synthesised Pt single-site complex sample at different working potentials for HER in $0.5 \mathrm{M} \mathrm{H}_{2} \mathrm{SO}_{4}$. The inset graphs show the energy shifts of $\mathrm{Pt} \mathrm{L}_{3}$ absorption edge (left) and white-line peak (right). d, The corresponding $\mathrm{K}^{2}$-weighted Fourier-transformed EXAFS spectra (magnitude component). The vertical dashed lines show the Pt-N (red), Pt-Cl (orange) and Pt-Pt (grey) peaks.

starting point for the analysis. The operando EXAFS data are best described by the structural model for trans-dianilinedichloroplatinum(II), $\left(\mathrm{C}_{6} \mathrm{H}_{5} \mathrm{NH}_{2}\right)_{2} \mathrm{Cl}_{2} \mathrm{Pt}(\mathrm{II})$, within the fitting range (K range: 3 to $12.5 \mathrm{~A}^{-1}, \mathrm{R}$ range: 1.1 to $2.5 \mathrm{~A}^{-1}$, misfit factor $0.8 \%$ ). This structure is similar to the previously reported crystal $\left(\mathrm{C}_{6} \mathrm{H}_{5} \mathrm{NH}_{2}\right)_{2} \mathrm{PtCl}_{2}$ complex, prepared by mixing aniline with $\mathrm{H}_{2} \mathrm{PtCl}_{6}$. $[45,46]$

Performing EXAFS curve-fitting analysis (platinum's first (1.5 to $2.1 \mathrm{~A}$ ) and second (2.3 to $3.1 \mathrm{~A})$ coordination shells) on the spectra collected at different potentials results in the curves in Figure $4 \mathrm{~b}$ and Table S1, which shows the average number of platinum, chlorine, and nitrogen atoms coordinated to each platinum atom (i.e. the coordination number), as a function of applied potential. Specifically, Figure 4c highlights the evolution of the EXAFS spectra while decreasing potential in the R-space fitted with the contributions of Pt-N, Pt-Cl and Pt-Pt paths. At OCV, the coordination number for both chlorine and nitrogen is about 2.5. As the potential is lowered, the coordination number for nitrogen decreases to about 1.0 by a potential of $0.1 \mathrm{~V}$ versus RHE. It continues to decrease to about 0.5 by $0 \mathrm{~V}$ (but before the onset of the HER). The chlorine coordination number stays higher than the nitrogen coordination number. This indicates that the aniline molecules dissociate from the complex before the chloride ions do, likely due to the more ionic character and higher bond energy of the Pt-Cl bonds compared to the Pt-N bonds. [47] The platinum coordination number increases as the potential is lowered, ultimately reaching a value 

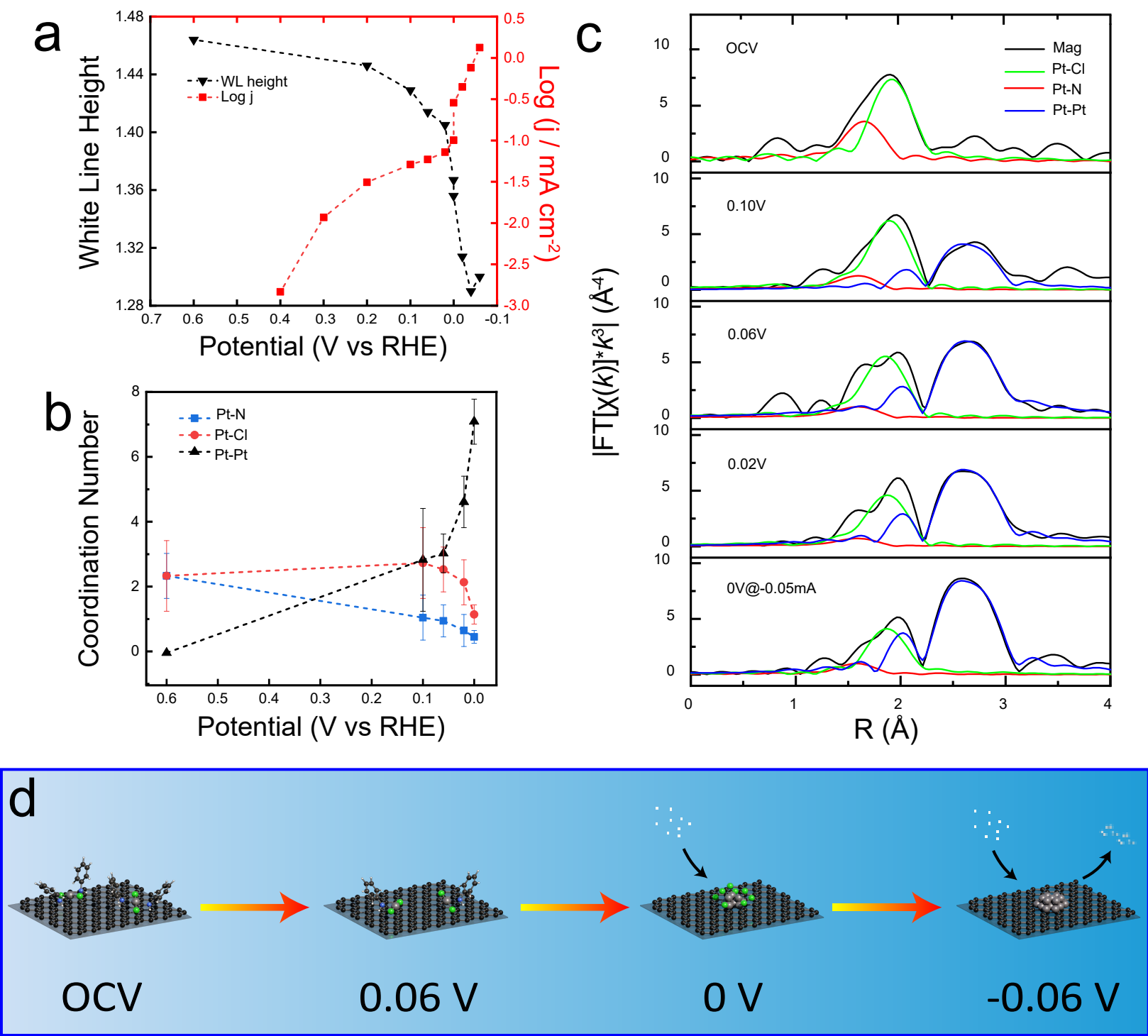

Figure 4. a, Amplitude of normalised white-line peak of $\mathrm{Pt} \mathrm{L}_{3}$-edge operando XANES spectra (black) and the corresponding catalytic activity (red) at different potentials. $\log (\mathrm{j})$ is calculated using relatively stable current density at the end of each $\mathrm{CA}$ test. $\mathbf{b}$, Coordination number of Pt-N, Pt-Cl and Pt-Pt fitting paths for operando EXAFS data of Pt single-site complex at selected potentials by $0 \mathrm{~V}$. Error bars in (b) represent the uncertainties of fitting results. c, Contribution of different paths $(\mathrm{Pt}-\mathrm{N}, \mathrm{Pt}-\mathrm{Cl}$ and $\mathrm{Pt}-\mathrm{Pt}$ ) to fit the corresponding EXAFS spectra. The details of the fitting results shown in K-space and R-space can be found in Supplementary Figure S11. d, Proposed schematic model for the evolution of (Pt/Ani/Ar) sample from single-site complex to subnanocluster based on the operando EXAFS analysis (grey, Pt; green, Cl; blue, N; black, carbon; white, $\mathrm{H}$ ). 
of about 7.0 by $0 \mathrm{~V}$ versus RHE. This indicates the agglomeration of platinum as the potential is lowered, which is also in line with the reduction of the oxidation state of platinum described in Figure 4a. [48]

Together these data elucidate how the platinum bonding environment in an SSC changes as a function of applied potential during operation, as described schematically in Figure 4d. As the potential is lowered from OCV, the dianilinedichloroplatinum(II) decomposes as the aniline dissociates from the platinum first, followed by the dissociation of the chloride ions. Simultaneously, the $5 d$ valence orbitals of platinum are filled. By the onset of the HER at $0 \mathrm{~V}$ versus RHE, the dianilinedichloroplatinum(II) complexes have completely dissociated, and the metallic platinum(0) single atoms agglomerate into nanoclusters which catalyse the HER.

\section{Conclusions}

In this work, we use operando, synchrotron X-ray absorption spectroscopy to characterize how the oxidation state and bonding environment of platinum changes in an SSC for the HER at the molecular level. First we show successful synthesis of a platinum SSC, confirming the platinum is atomically dispersed by STEM and XRD. We observe a mixture of platinum(II) and platinum(IV) by XPS and XANES, and we confirm platinum coordination to aniline by ex situ EXAFS. We then probe the oxidation state and molecular structure as a function of applied potential using operando EXAFS and XANES with synchrontron radiation. We find that as the potential is lowered from OCV the platinum oxidation state decreases, breaking bonds between platinum atoms and aniline ligands first. The $\mathrm{Pt}-\mathrm{Cl}$ bonds also break, but to a lesser extent than the Pt-N bonds. Finally, once the onset of the HER at $0 \mathrm{~V}$ versus RHE is reached, the platinum is completely reduced and metallic platinum atoms agglomerate to form catalytic nanoclusters. Our work suggests that many catalysts that achieve atomically dispersed platinum by aniline coordination do not remain atomically dispersed during device operation. This complicates the interpretation of many experimental results. Furthermore, this operando study establishes two important structure-property relationship for aniline-complex platinum SSCs. First, platinum exists as metallic platinum (that is, in the oxidation state 0) while catalysing the HER. Second, the molecular structure of the platinum complexes is lost by the onset of the HER. These findings are an important contribution to atomic understanding of structural evolution of SSCs for the HER and the development of high-performance platinum SSCs for clean hydrogen production.

\section{Declaration of interest}

The authors declare no competing interests.

\section{Data availability}

The authors declare that all data supporting the findings of this study are included within the paper and its Supplementary Information files. Source data are available from the corresponding author upon reasonable request.

\section{Acknowledgements}

The authors would like to acknowledge the financial support of the Jiangsu Industrial Technology Research Institute (JITRI) and the Henry Royce Institute (through UK Engineering and Physical Science Research Council grant EP/R010145/1) for capital equipment. We acknowledge Diamond Light Source for time on B18 Beamline under Proposal SP-26066. We are grateful to the David Cockayne Centre for Electron Microscopy for the use of their electron microscopes.

\section{Author contributions}

P.T and M.P conceived and designed the experiments. P.T prepared the samples and performed the electrochemical tests. P.T, J.C and J.H.W performed the aberration-corrected STEM characterisation and analysed the data. D.G and R.A conducted the operando XAFS experiments at Diamond Light Source under the proposal of SP_26066. P.T and H.J.L analysed the operando XAFS data. P-Y.H and P.T prepared reference samples and calibrated the three-electrode cell used in operando XAFS study. S.N performed the XPS characterisation and contributed to XPS data interpretation. K.H conducted the DFT calculations. P.T, K.H and C.W wrote the manuscript with input 
from all authors. M.P. supervised the design of the project and gave frequent, in-depth scientific input.

\section{Experimental procedures}

\section{Chemicals}

Chloroplatinic acid hexahydrate $(37.5 \% \mathrm{Pt}$ basis $)$, aniline $(99.5 \%)$ and ammonium persulfate $(98 \%)$ were purchased from Sigma-Aldrich. Ultra-pure water (18.2 M $\Omega$ cm-1; by Direct-Q Water Purification System) was used for the electrochemical tests.

\section{Synthesis of Pt single-atom/clusters at surface of graphene}

The Pt single-site complex catalysts stabilised on the surface of thin-layer graphene by aniline molecules was prepared using a modified impregnation method. [10] Firstly, thin-layer graphene is grown by a CVD method, and transferred onto the surface of well-polished glassy carbon (GC). [49] Then, graphene surface is cleaned by annealing under $\mathrm{Ar}$ $(70 \mathrm{sccm}) / \mathrm{H}_{2}(5 \mathrm{sccm})$ flow at $250{ }^{\circ} \mathrm{C}$ for 2 hours. The remaining sulphur from ammonium persulfate and small organics are removed. Then samples with different Pt structures are synthesised on the clean surface of graphene. Sample one (Pt/Ani/Ar): $4 \mu \mathrm{L}$ of aniline drop casted on a $1 \mathrm{~cm}^{2}$ graphene nanosheets, dried in ambient conditions for 15 minutes, then drop casting $10 \mu \mathrm{L}$ of $10 \mathrm{mM} / \mathrm{L}$ $\mathrm{H}_{2} \mathrm{PtCl}_{6}$ ethanol solution. It is then annealed in argon atmosphere at $100{ }^{\circ} \mathrm{C}$ for $1.5 \mathrm{~h}$. Sample two $(\mathbf{P t} / \mathbf{A r})$ : synthesised using the same procedure without drop casting aniline. For further comparison, samples are prepared by annealing $(\mathrm{Pt} / \mathrm{Ani} / \mathrm{Ar})$ at $200{ }^{\circ} \mathrm{C}$ instead of $100{ }^{\circ} \mathrm{C}\left(\mathbf{P t} / \mathbf{A n i} / \mathbf{A r} / \mathbf{2 0 0}{ }^{\circ} \mathbf{C}\right)$, and replacing argon annealing condition with hydrogen annealing condition $\left(\mathbf{P t} / \mathbf{A n i} / \mathbf{H}_{\mathbf{2}}\right)$. After the annealing treatment, all samples are sequentially washed with $0.5 \mathrm{M}$ sulfuric acid then deionized water, and dried under vacuum overnight.

\section{Ex-situ STEM and XRD}

Room-temperature annular dark-field scanning transmission electron microscopy was performed with a JEOL ARM200F at an accelerating voltage of $80 \mathrm{kV}$. Dwell times of 10-20 $\mathrm{\mu s}$, a beam current of $35 \mathrm{pA}$, and a pixel size of $0.006 \mathrm{~nm} \mathrm{px}{ }^{-1}$ were used for imaging, with a convergence semi-angle of $25.5 \mathrm{mrad}$ and collecting inner-outer angles 68 to $275 \mathrm{mrad}$. XRD patterns were collected using a monochromated $\mathrm{Cu} \mathrm{K \alpha} \mathrm{X}$-ray source from $5^{\circ}$ to $90^{\circ}(2 \vartheta)$, at a scan rate of $5^{\circ} \mathrm{min}^{-1}$.

\section{Ex-situ XPS and XAS}

X-ray photoemission spectroscopy was conducted using a PHI VersaProbe III system generating monochromatic Al X-rays at $1486 \mathrm{eV}$. Survey scans and high-resolution elemental scans were acquired at pass energies of $224 \mathrm{eV}$ and $55 \mathrm{eV}$ respectively. The binding energies were calibrated using the C 1s line at $284.8 \mathrm{eV}$ as reference. The XPS spectra were fit using Gaussian-Lorentzian and Voigt lineshapes (where asymmetry was pronounced), and quantified with the help of CasaXPS software. X-ray absorption fine structure (XAFS) measurements at the $\mathrm{Pt} \mathrm{L}_{3}$ edge were carried out at the B18 Core EXAFS beamline of Diamond Light Source. The Pt reference samples $(300 \mathrm{mg}$ ) were finely ground, mixed homogeneously with five parts of cellulose and pressed into $13 \mathrm{~mm}$ diameter pellets. Commercial $\left(\mathrm{NH}_{4}\right)_{2} \mathrm{PtCl}_{6}$ (Sigma-Aldrich, 99.9\%), $\mathrm{PtCl}_{2}$ (Sigma-Aldrich, $99.9 \%$ ) were used as references. All spectra were recorded in transmission mode at room temperature.

\section{Electrochemical measurements}

The catalytic activity of synthesised samples were evaluated under a three-electrode cell with graphite rod as counter electrode and $\mathrm{Ag} / \mathrm{AgCl}$ (in $3 \mathrm{M} \mathrm{KCl}$ ) as reference electrode in $0.5 \mathrm{M} \mathrm{Ar} / \mathrm{N}_{2}$-purged $\mathrm{H}_{2} \mathrm{SO}_{4}$ solution. A $0.2 \mathrm{~mm}$ thick well-polished glassy carbon plate (Sigma-Aldrich Company Ltd.) was firstly used to transfer and support the thin-layer graphene nanosheets. Then the GC supporting well-dispersed Pt catalysts with $(1 \mathrm{~cm} \times 1 \mathrm{~cm})$ defined area was used as the working electrode. A metal clip was used to connect the working electrode with an external circuit. The chronoamperometric performances were recorded by a Ivium Technologies or Biologic VMP3 potentiostat. All potentials were calibrated versus RHE using $\mathrm{E}(\mathrm{RHE})=\mathrm{E}(\mathrm{Ag} / \mathrm{AgCl})+0.197 \mathrm{~V}+$ $0.0592 \times \mathrm{pH}$.

\section{Operando XAS}

EXAFS and XANES were performed at the B18 Core EXAFS beamline of Diamond Light Source. The measurements were carried out using the Cr-coated 
branch of collimating and focusing mirrors, and a $\mathrm{Si}(111)$ double-crystal monochromator. The size of the beam at the sample position was ca. $1 \mathrm{~mm}$ $(\mathrm{h}) \times 1 \mathrm{~mm}(\mathrm{v})$. Samples were measured both in static electrolyte and operando conditions. The data were collected in fluorescence mode, by means of a 36-element solid state germanium detector, the ion chamber at the front of the sample has been used for measurement of incoming photons ( $\mathrm{I}_{0}$ filled with a mixture of $80 \mathrm{mbar}$ of $\mathrm{Ar}$ and 1020 mbar of $\mathrm{He}$ to optimise sensitivity at $15 \%$ efficiency). An electrochemical cell adapted to the B18 beamline of the UK's Synchrotron Diamond Light Source was designed for the operando study shown in Supplementary Figure S8. Before the operando electrochemical measurements, the $\mathrm{Pt} \mathrm{L}_{3}$ edge spectra were measured on contact with the liquid electrolyte. The operando XAFS spectra of the $\mathrm{Pt} \mathrm{L}_{3}$ edge $(11564 \mathrm{eV})$ were obtained from 200 $\mathrm{eV}$ before the edge up to $750 \mathrm{eV}$ after the edge (corresponding to $14 \AA^{-1}$ in k-space). The measuring time was 2 minutes per spectrum. When indicated, 8 repetitions at each potential were acquired and then merged to obtain a better signal to noise ratio. Data were normalised using the Athena [50] program with a linear pre-edge and polynomial post-edge background subtracted from the raw data. EXAFS fits were performed using ARTEMIS software. [50]

\section{Computational methods}

DFT calculations were performed using the Vienna ab initio Simulation Package (VASP) [51, 52]. We performed $\Gamma$-point only calculations using a plane-wave kinetic-energy cutoff of $520 \mathrm{eV}$ with electronic and ionic convergence criteria of $10^{-5} \mathrm{eV}$ and $0.05 \mathrm{eV} \AA^{-1}$, respectively. The Perdew-Burke-Ernzerhof (PBE) functional was used for all structures.

\section{References}

1. Hydrogen From Renewable Power: Technology outlook for the energy transition (IRENA, 2018).

2. Lagadec, M. F. \& Grimaud, A. Water electrolysers with closed and open electrochemical systems. Nature Materials 19, 1140-1150 (2020).
3. McCrory, C. C. et al. Benchmarking hydrogen evolving reaction and oxygen evolving reaction electrocatalysts for solar water splitting devices. Journal of the American Chemical Society 137, 4347-4357 (2015).

4. Cui, X., Li, W., Ryabchuk, P., Junge, K. \& Beller, M. Bridging homogeneous and heterogeneous catalysis by heterogeneous single-metal-site catalysts. Nature Catalysis 1, 385-397 (2018).

5. Kibsgaard, J. \& Chorkendorff, I. Considerations for the scaling-up of water splitting catalysts. Nature Energy 4, 430-433 (2019).

6. Qiao, B. et al. Single-atom catalysis of $\mathrm{CO}$ oxidation using $\mathrm{Pt}_{1} / \mathrm{FeO}_{\mathrm{x}}$. Nature chemistry $\mathbf{3}$, 634-641 (2011).

7. Cheng, N., Zhang, L., Doyle-Davis, K. \& Sun, X. Single-atom catalysts: from design to application. Electrochemical Energy Reviews 2, 539-573 (2019).

8. Yang, X.-F. et al. Single-atom catalysts: a new frontier in heterogeneous catalysis. Accounts of chemical research 46, 1740-1748 (2013).

9. Wang, A., Li, J. \& Zhang, T. Heterogeneous single-atom catalysis. Nature Reviews Chemistry 2, 65-81 (2018).

10. Ye, S. et al. Highly stable single Pt atomic sites anchored on aniline-stacked graphene for hydrogen evolution reaction. Energy $\&$ Environmental Science 12, 1000-1007 (2019).

11. Xu, H., Cheng, D., Cao, D. \& Zeng, X. C. A universal principle for a rational design of single-atom electrocatalysts. Nature Catalysis 1, 339-348 (2018).

12. Chen, Y. et al. Single-atom catalysts: synthetic strategies and electrochemical applications. Joule 2, 1242-1264 (2018).

13. Thomas, J. M., Raja, R. \& Lewis, D. W. Single-site heterogeneous catalysts. Angewandte Chemie International Edition 44, 6456-6482 (2005).

14. Pelletier, J. D. \& Basset, J.-M. Catalysis by design: well-defined single-site heterogeneous catalysts. Accounts of chemical research 49, 664-677 (2016). 
15. Zhao, D. et al. Atomic site electrocatalysts for water splitting, oxygen reduction and selective oxidation. Chemical Society Reviews 49, 2215-2264 (2020).

16. Wang, J., Dou, S. \& Wang, X. Structural tuning of heterogeneous molecular catalysts for electrochemical energy conversion. Science Advances 7, eabf3989 (2021).

17. Li, X., Yang, X., Zhang, J., Huang, Y. \& Liu, B. In Situ/Operando techniques for characterization of single-atom catalysts. ACS Catalysis 9, 2521-2531 (2019).

18. Maurer, F. et al. Tracking the formation, fate and consequence for catalytic activity of Pt single sites on $\mathrm{CeO}_{2}$. Nature Catalysis 3, 824-833 (2020).

19. Fang, S. et al. Uncovering near-free platinum single-atom dynamics during electrochemical hydrogen evolution reaction. Nature communications 11, 1029 (2020).

20. Kaiser, S. K. et al. Nanostructuring unlocks high performance of platinum single-atom catalysts for stable vinyl chloride production. Nature Catalysis 3, 376-385 (2020).

21. Ren, Y. et al. Unraveling the coordination structure-performance relationship in $\mathrm{Pt}_{1} / \mathrm{Fe}_{2} \mathrm{O}_{3}$ single-atom catalyst. Nature communications 10, 4500 (2019).

22. Fei, H. et al. Single atom electrocatalysts supported on graphene or graphene-like carbons. Chemical Society Reviews 48, 5207-5241 (2019).

23. Lee, J. K. et al. Metal atom markers for imaging epitaxial molecular self-assembly on graphene by scanning transmission electron microscopy. ACS Nano 13, 7252-7260 (2019).

24. Li, Y. et al. An oxygen reduction electrocatalyst based on carbon nanotube-graphene complexes. Nature Nanotechnology 7, 394-400 (2012).

25. Li, X. et al. Precursor design for high density single Pt atom sites on MoS2: enhanced stability at elevated temperatures and reduced 3D clustering. Chemistry of Materials 32, 2541-2551 (2020).

26. Shiell, T. B. et al. In situ analysis of the structural transformation of glassy carbon under compression at room temperature. Physical Review B 99, 024114 (2019).
27. Yang, M. et al. A common single-site $\mathrm{Pt}(\mathrm{II})-\mathrm{O}(\mathrm{OH}) \mathrm{x}$ - species stabilized by sodium on "active" and "inert" supports catalyzes the water-gas shift reaction. Journal of the American Chemical Society 137, 3470-3473 (2015).

28. Liu, J. et al. High performance platinum single atom electrocatalyst for oxygen reduction reaction. Nature communications 8, 15938 (2017).

29. Cheng, X. et al. Highly active, stable oxidized platinum clusters as electrocatalysts for the hydrogen evolution reaction. Energy and Environmental Science 10, 2450-2458 (2017).

30. Imaoka, T. et al. Platinum clusters with precise numbers of atoms for preparative-scale catalysis. Nature Communications 8, 688 (2017).

31. Lim, T. et al. Atomically dispersed $\mathrm{Pt}-\mathrm{N}_{4}$ sites as efficient and selective electrocatalysts for the chlorine evolution reaction. Nature Communications 11, 412 (2020).

32. Li, X. et al. Single-atom Pt as co-catalyst for enhanced photocatalytic $\mathrm{H}_{2}$ evolution. Advanced Materials 28, 2427-2431 (2016).

33. Obreja, L., Foca, N., Popa, M. \& Melnig, V. Alcoholic reduction platinum nanoparticles synthesis by chemical method, biomaterials in biophysics. Medical Physics and Ecology (2008).

34. Wang, J. et al. In Situ identifying the dynamic structure behind activity of atomically dispersed Platinum catalyst toward hydrogen evolution reaction. Small 17, 2005713 (2021).

35. Cao, L. et al. Identification of single-atom active sites in carbon-based cobalt catalysts during electrocatalytic hydrogen evolution. Nature Catalysis 2, 134-141 (2019).

36. Ghanim, A. H. et al. Low-loading of $\mathrm{Pt}$ nanoparticles on 3D carbon foam support for highly active and stable hydrogen production. Frontiers in chemistry 6, 523 (2018).

37. Kang, J. et al. Platinum Atoms and Nanoparticles Embedded Porous Carbons for Hydrogen Evolution Reaction. Materials 13, 1513 (2020). 
38. Mansour, A., Cook Jr, J. \& Sayers, D. Quantitative technique for the determination of the number of unoccupied d-electron states in a platinum catalyst using the $\mathrm{L}_{2,3} \mathrm{X}$-ray absorption edge spectra. The Journal of Physical Chemistry 88, 2330-2334 (1984).

39. Horch, S. et al. Enhancement of surface self-disfusion of platinum atoms by adsorbed hydrogen. Nature 398, 134-136 (1999).

40. Parkinson, G. S. et al. Carbon monoxide-induced adatom sintering in a $\mathrm{Pd}_{-} \mathrm{Fe}_{3} \mathrm{O}_{4}$ model catalyst. Nature Materials 12, 724-728 (2013).

41. Dessal, C. et al. Atmosphere-dependent stability and mobility of catalytic Pt single atoms and clusters on $\gamma-\mathrm{Al}_{2} \mathrm{O}_{3}$. Nanoscale 11, 6897-6904 (2019).

42. Shi, Y. et al. Electronic metal-support interaction modulates single-atom platinum catalysis for hydrogen evolution reaction. Nature communications 12, 3021 (2021).

43. Wang, J. et al. Redirecting dynamic surface restructuring of a layered transition metal oxide catalyst for superior water oxidation. Nature Catalysis 4, 212-222 (2021).

44. Malta, G. et al. Identification of single-site gold catalysis in acetylene hydrochlorination. Science 355, 1399-1403 (2017).

45. Mohanraju, K., Lee, H. \& Kwon, O. J. High loading $\mathrm{Pt}$ core/carbon shell derived from platinum-aniline complex for direct methanol fuel cell application. Electroanalysis 30, 1596-1601 (2018).

46. Kong, P. C. \& Rochon, F. D. Cis- and trans-platinum complexes of anilines. A study of isormerization reactions 1982.

47. Astakhov, A., Soliev, S. \& Chernyshev, V. Metal-ligand bond dissociation energies in the $\mathrm{Ni}, \mathrm{Pd}$, and Pt complexes with N-heterocyclic carbenes: effect of the oxidation state of the metal $(0,+2)$. Russian Chemical Bulletin 69, 2073-2081 (2020).

48. Hao, X. et al. Direct measurement and modulation of single-molecule coordinative bonding forces in a transition metal complex. Nature communications 4, 2121 (2013).
49. Fan, Y., He, K., Tan, H., Speller, S. \& Warner, J. H. Crack-free growth and transfer of continuous monolayer graphene grown on melted copper. Chemistry of Materials 26, 4984-4991 (2014).

50. Ravel, B. \& Newville, M. ATHENA, ARTEMIS, HEPHAESTUS: data analysis for X-ray absorption spectroscopy using IFEFFIT. Journal of synchrotron radiation 12, 537-541 (2005).

51. Theory of the crystal structures of selenium and tellurium: The effect of generalized-gradient corrections to the local-density approximation. Physical Review B 50, 13181-13185 (1994).

52. Kresse, G. \& Furthmüller, J. Efficiency of ab-initio total energy calculations for metals and semiconductors using a plane-wave basis set. Computational Materials Science 6, 15-50 (1996). 